\title{
A new design approach to innovative spectrometers. Case study: TROPOLITE.
}

\author{
Jean-Baptiste Volatier ${ }^{a}$, Stefan Baümer ${ }^{a}$, Bob Kruizinga ${ }^{a}$, Rob Vink $^{a}$ \\ ${ }^{a}$ TNO, Stieltjesweg 1, Delft, Netherlands;
}

\begin{abstract}
Designing a novel optical system is a nested iterative process. The optimization loop, from a starting point to final system is already mostly automated. However this loop is part of a wider loop which is not. This wider loop starts with an optical specification and ends with a manufacturability assessment.

When designing a new spectrometer with emphasis on weight and cost, numerous iterations between the optical- and mechanical designer are inevitable. The optical designer must then be able to reliably produce optical designs based on new input gained from multidisciplinary studies.

This paper presents a procedure that can automatically generate new starting points based on any kind of input or new constraint that might arise. These starting points can then be handed over to a generic optimization routine to make the design tasks extremely efficient. The optical designer job is then not to design optical systems, but to meta-design a procedure that produces optical systems paving the way for system level optimization.

We present here this procedure and its application to the design of TROPOLITE a lightweight push broom imaging spectrometer.
\end{abstract}

Keywords: optical design, imaging spectrometer, starting point, yybar diagram, plate diagrams, optimization

\section{TROPOLITE}

\subsection{Scope}

A push broom imaging spectrometer for earth observation contains six functional optical elements as shown in figure 1. Its measurement principle is described in figure 2. In this study we focus on investigating allreflective solutions for the ensemble collimator, imager for a UVIS channel (320-495nm). It is common to use all-reflective telescope designs, ${ }^{1}$ however the ensemble collimator, imager is commonly realized via several channels of catadioptrics systems.

Advantages of all-reflective solutions are:

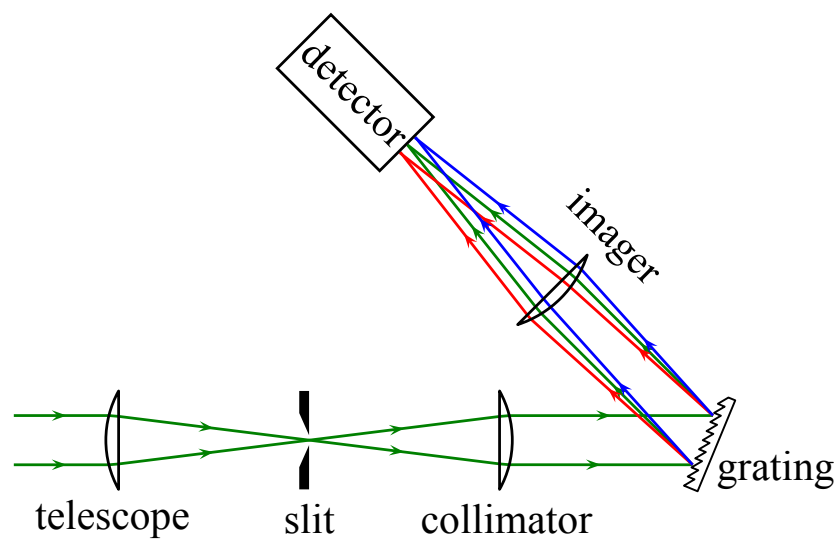

Figure 1. Functional layout of a push broom spectrometer in spectral plane. Optical elements are represented as lenses for sake of clarity. The slit is rectangular such as imaging a line on earth (the longer slit dimension is out-of-plane in the figure coordinate system). The image on detector is two dimensional with one dimension corresponding to sampling locations on earth (out-of-plane in the figure) and the other dimension corresponding to sampling locations in spectrum. 


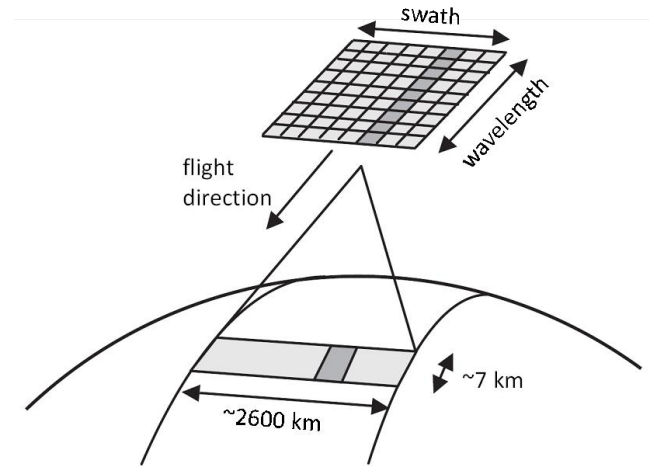

Figure 2. Measurement principle of a push-broom imaging spectrometer. ${ }^{1}$

- Design can be copied unchanged for other channels (with the exception of the grating).

- Fewer optical elements due to the insensitivity to chromatic aberrations.

- More design freedom regarding stray light baffling due to the off-axis nature of such a design.

This can lead to a lighter and cheaper instrument.

\subsection{Requirements}

Table 1. Driving requirements.

\begin{tabular}{|llcl|}
\hline F\# & $\begin{array}{l}\text { spectral } \\
\text { field }\end{array}$ & 16 & \\
& spectral & 0.1 & $\mathrm{~mm}$ \\
& field & 24.0 & $\mathrm{~mm}$ \\
\hline spot size (RMS) & & 0.013 & $\mathrm{~mm}$ \\
\hline co-registration & & 0.003 & $\mathrm{~mm}$ \\
\hline Max Volume & & 1.5 & $\mathrm{~L}$ \\
\hline
\end{tabular}

The co-registration is defined as the peak to valley straightness of the image at detector plane of a point on earth. The volume requirement is the most stringent and was the motivation to explore new methods capable of finding compatible starting points.

\section{NEW DESIGN APPROACH}

\subsection{Theoretical background}

Our new design approach makes use of well known techniques such as $y-\bar{y}$ diagrams, ${ }^{2}$ Seidel aberration theory and plate diagrams ${ }^{3}$ that we revive by combining them together, and with modern computing technology.

The link between $y-\bar{y}$ diagrams and Seidel aberrations is not new ${ }^{4}$ but we will also show that unsurprisingly $y-\bar{y}$ and plate diagrams are also connected.

\subsection{1 $y-\bar{y}$ diagram}

A $y-\bar{y}$ diagram is a paraxial representation of an optical system. It consists of the projection of an off-axis ray, marginal in the sagittal plane ("skew" ray see figure 3) on the image plane as shown in figure 4.

$y-\bar{y}$ diagrams have many interesting properties. The most important ones for our following discussion are: 


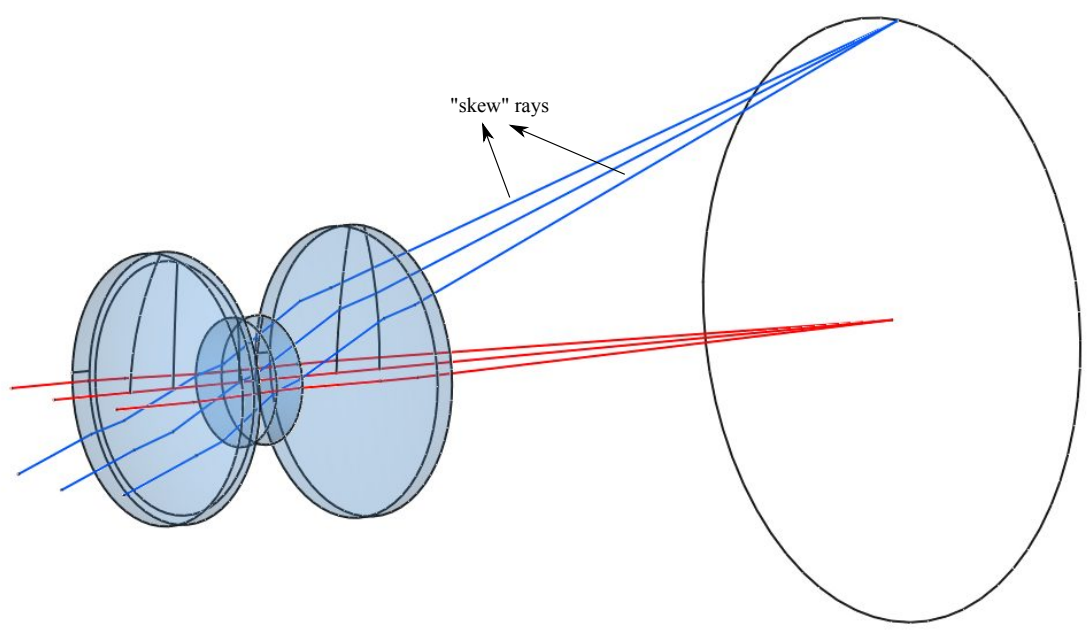

Figure 3. The famous Cook achromatic triplet, seen in perspective with only sagittal rays drawn for two field points. The two "skew" rays are indicated.

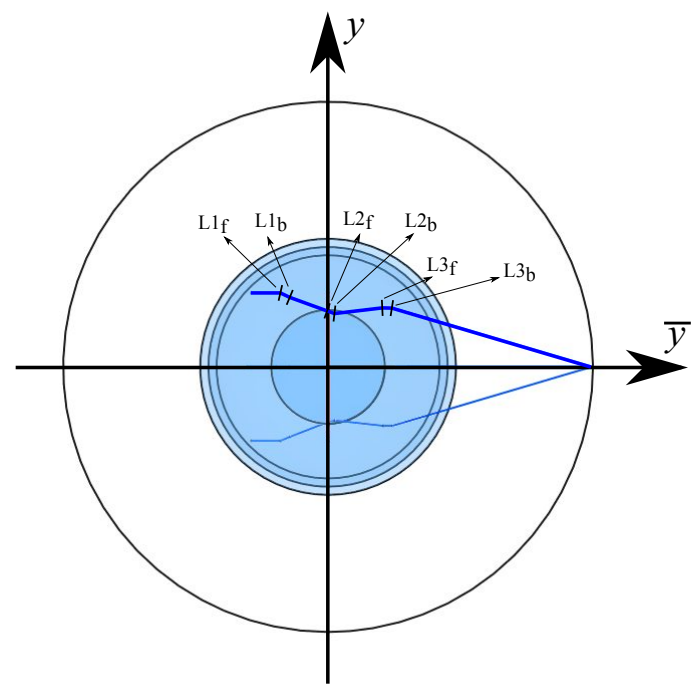

Figure 4. This is the same system as in figure 3, seen from the back and rotated around its optical axis (z). The projection on image plane of one of these "skew" rays is a $y-\bar{y}$ diagram. Each change of direction (indicated by a vertical mark) corresponds to an optical surface. We choose one of the rays out of the two in order to fulfill the convention that $y-\bar{y}$ diagrams propagate in the clockwise direction. 


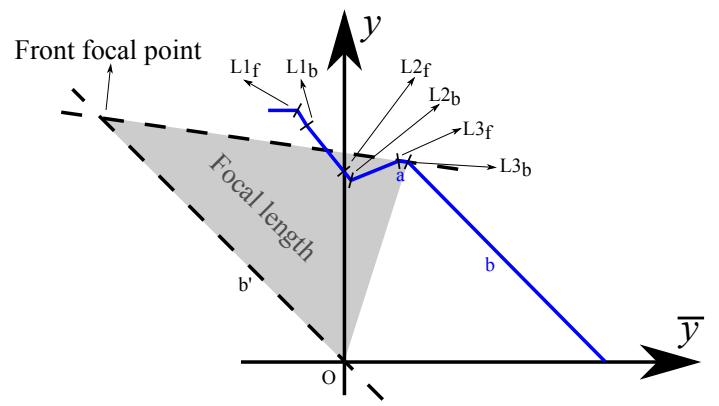

Figure 5. This is the same diagram as in figure 4, only rescaled along the $y$ axis for better clarity. The area of the filled triangle is proportional to the focal length of the last optical surface.

- Points on the rays in the diagram and on a line through the origin represent conjugated planes.

- Areas of triangles consisting of two points on the same ray segment and the origin are proportional to distances along the optical axis.

- $|y|+|\bar{y}|$ at a point is equal to the beam size at the optical plane represented by that point.

The proportionality constant linking triangle areas and distances is:

$$
d=\frac{2 n S}{H}
$$

Where :

- $d$ is the distance

- $n$ is the refractive index

- $S$ is the area of the triangle

- $H$ is the lagrange invariant.

The Lagrange invariant is defined as (on the i-th plane) :

$$
H=n \bar{u}_{i} y_{i}-n u_{i} \bar{y}_{i}
$$

And :

$$
\begin{aligned}
\bar{u}_{i} & =\frac{y_{i+1}-\overline{y_{i}}}{d_{i, i+1}} \\
u_{i} & =\frac{y_{i+1}-y_{i}}{d_{i, i+1}}
\end{aligned}
$$

Where $d_{i, i+1}$ is the distance between the two planes $i$ and $i+1$ along the optical axis and $y_{i}-\overline{y_{i}}$ are the $y-\bar{y}$ coordinates of the plane $i$. For a particular system, $H$ is evaluated once at the most convenient plane, usually object/image or pupil.

In figure 5 we construct the front focal point of the back surface of lens 3 by :

Proc. of SPIE Vol. 9131 91310L-4 
1. Drawing a line parallel to the exit ray (noted $b$ in figure 5) and going through the origin (noted $O$ ). Any point on that line is conjugated to infinity in $L 3_{b}$ image space.

2. The intersection of that line and the entrance ray of $L 3_{b}$ (noted $a$ ) is then representative of an optical plane in $L 3_{b}$ object space conjugated to infinity in $L 3_{b}$ image space.

3. The area of the triangle limited by $L 3_{b}$, its front focal point and $O$ represents the focal length of $L 3_{b}$.

4. From the refractive index of lens 3 we can even determine $L 3_{b}$ curvature.

\subsubsection{Seidel aberrations}

The Seidel aberrations $\operatorname{are}^{5}$ :

$$
\begin{array}{lr}
s_{1}=y A^{2} \Delta\left(\frac{u}{n}\right) & \text { Spherical aberration } \\
s_{2}=y A \bar{A} \Delta\left(\frac{u}{n}\right) & \text { Coma } \\
s_{3}=y \bar{A}^{2} \Delta\left(\frac{u}{n}\right) & \text { Astigmatism } \\
s_{4}=-c H^{2} \Delta\left(\frac{1}{n}\right) & \text { Field curvature }
\end{array}
$$

Where :

$$
\begin{aligned}
& A=n(u+c y) \\
& \bar{A}=n(\bar{u}+c \bar{y})
\end{aligned}
$$

$c$ is the surface curvature. We then see that $y-\bar{y}$ diagrams provides all the information that we need to evaluate Seidel aberrations.

\subsubsection{Plate diagrams}

Plate diagrams also known as see-saw diagrams provide a way to characterize third order aberrations. It consists of mapping the locations of the equivalent Schmidt plate for each optical surface in a common space (by convention object space). ${ }^{3}$ Equivalent Schmidt plates are plates with a $4^{\text {th }}$ order polynomial sag on one side such that it completely compensates the spherical aberration of an optical surface. Schmidt plates are located at planes conjugated with the center of curvature of each optical surface.

Finding these planes is easy with a $y-\bar{y}$ diagram and is described in figure 7 for the example telescope of figure 8 .

1. We prolongate the segment $r_{2}$ to find the intersection with the parallel of $r_{3}$ going through $O$, in this case this is the $y$ axis. That way we found the front focal plane (similar to figure 5).

2. The front focal point is equidistant of M2 and the center of curvature. The center of curvature is also on the line prolongation of $r_{2}$.

3. The line going through $O$ and $\mathrm{CoC}$ intersect $r_{1}$ which represents the object space. At this intersection is therefore the $\mathrm{CoC}$ conjugate in object space. 


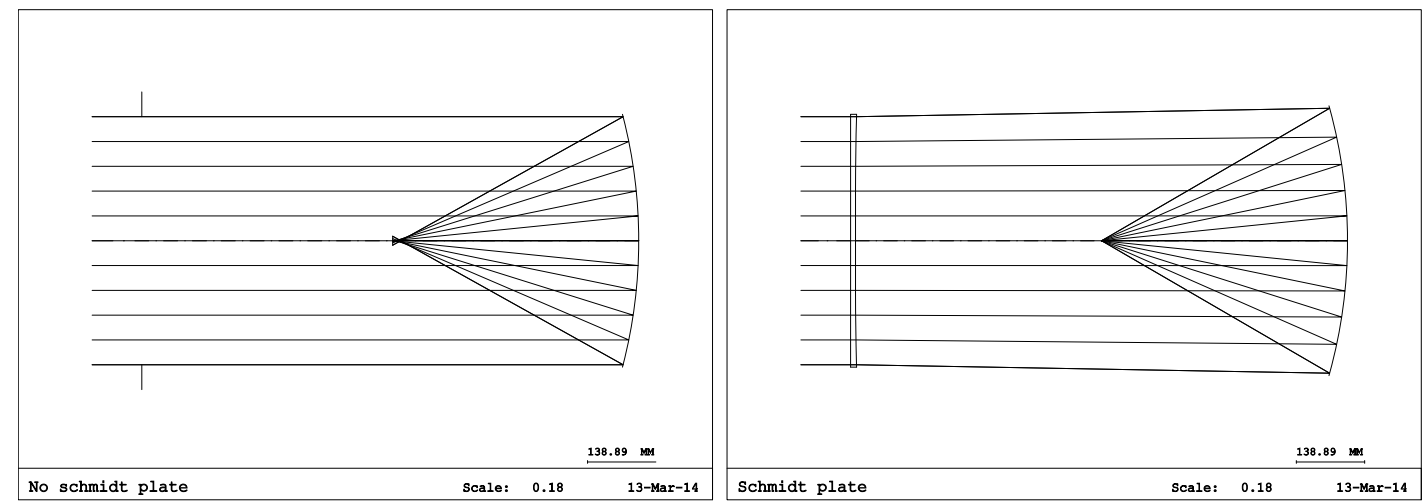

Figure 6. On the left a spherical mirror without Schmidt corrector plate. One can see that it exhibits significant spherical aberration. On the right the same mirror but with a Schmidt corrector plate located at its center of curvature. The spherical aberration is totally corrected.

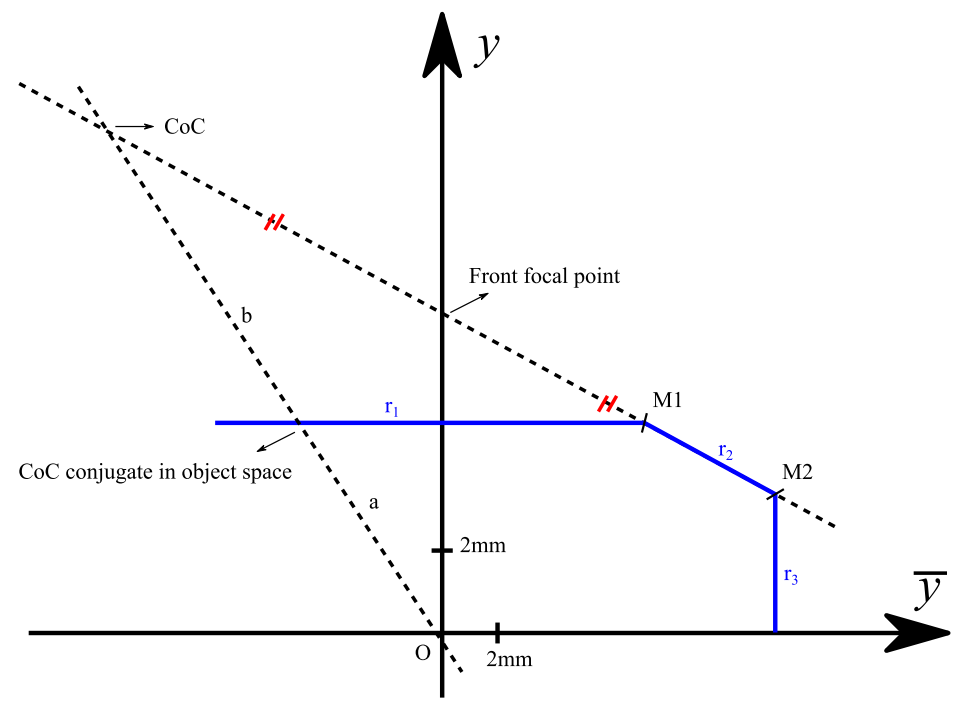

Figure 7. $y-\bar{y}$ diagram of an example telescope. $b$ is the length of the segment $O-$ CoC. $a$ is the length of the segment $O$ - CoC conjugate in object space.

Then the performance of the system is described as:

$$
\begin{aligned}
\text { Spherical Aberration } & =\sum W_{i} \\
\text { Coma } & =\sum \overrightarrow{r_{i}} W_{i} \\
\text { Astigmatism } & =\sum{\overrightarrow{r_{i}}}^{2} W_{i}
\end{aligned}
$$

Where $W_{i}$ is the retardation of each Schmidt plate (proportional as $s_{1}$ see (6)) and $\overrightarrow{r_{i}}$ is the location of the Schmidt plate with respect to the entrance pupil.

Using $\frac{b}{a}$ as seen in figure 7 we can compute the $y_{i}$ component of $\overrightarrow{r_{i}}$ when we use a mirror off-axis. As it links the displacement of the center of curvature with the displacement of the equivalent plate, $\frac{b}{a}$ is together with $W_{i}$ a measure of how sensitive the mirror is to off-axis angles. This is of importance for the designer when choosing a good starting point.

The results of such a description are summarized in table 2 for the example telescope drawn in 8 . Figure 9 shows us that this system will have significant aberration however it also shows us that this folding layout is 


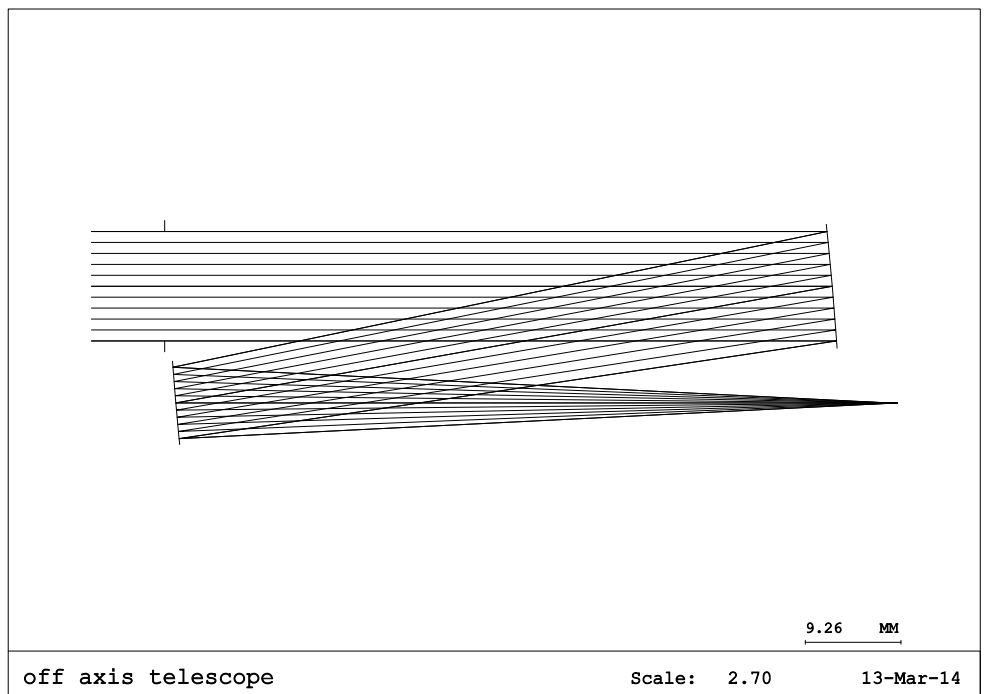

Figure 8. Example of an off-axis telescope corresponding to the $y-\bar{y}$ in figure 7 .

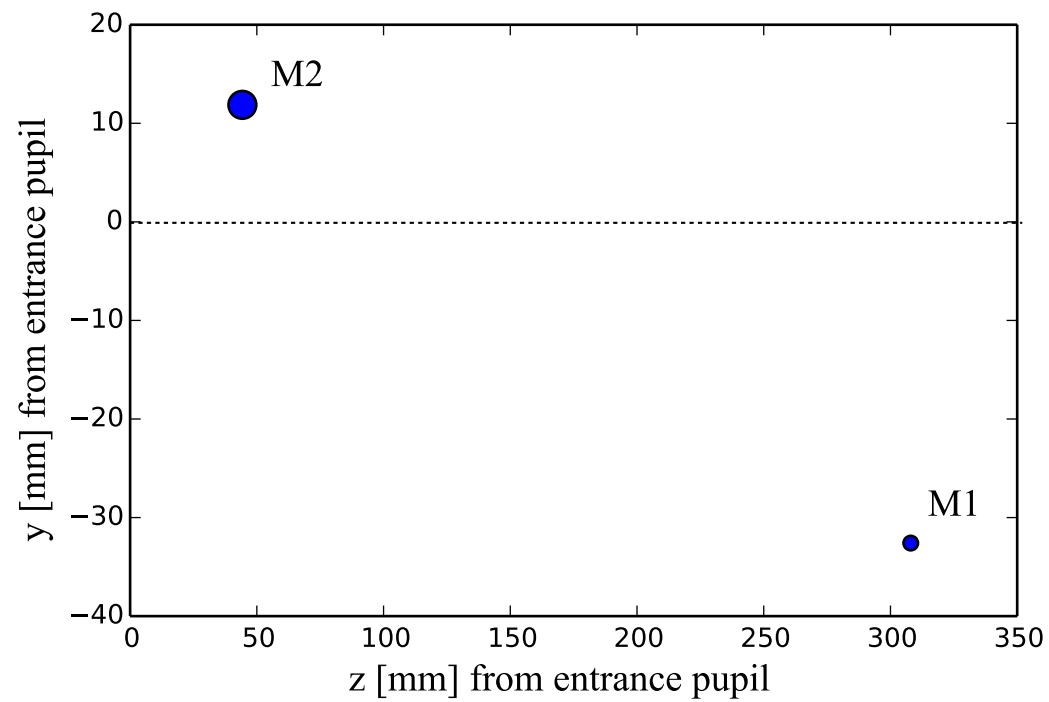

Figure 9. Plate diagram of the example telescope.

Proc. of SPIE Vol. 9131 91310L-7 
Table 2. Relevant data for the example telescope. $z_{i}$ and $y_{i}$ are the two components of $\overrightarrow{r_{i}}$. SA is the spherical aberration introduced by each surface following the CodeV normalization convention.

\begin{tabular}{|ccccccc|}
\hline & radius & $z_{i}$ & $\frac{b}{a}$ & off-axis angle & $y_{i}$ & SA \\
\hline M1 & 372 & 308 & 1 & 5 & -33 & -0.0003 \\
\hline M2 & 327 & 44 & 0.42 & -5 & 12 & -0.0011 \\
\hline
\end{tabular}

favored with respect to coma.

\subsection{Exploration of the solution space}

\subsubsection{Brute force search}

TROPOLITE is a lightweight spectrometer, meaning that one of the driving requirement is volume, which should be under 1.5L. Using $y-\bar{y}$ we can estimate the volume of each solution by :

$$
V=\operatorname{MAX}\left(T_{i}\right) \sum\left(\left|y_{i}\right|+\left|\bar{y}_{i}\right|\right)
$$

Where $T_{i}$ is the distance along the optical axis after the i-th element.

We can estimate the on-axis performance using Seidel sums. However we are going to use these systems off-axis so we will also look at $\frac{b}{a}$ as an indication of how sensitive the system is going to be and also at the Seidel contributions of each element. We have put the formalism of $y-\bar{y}$, Seidel and plate diagrams in a computer program that is able to evaluate a potential solution in a matter of microseconds on a laptop (Intel Core i7 at $2.4 \mathrm{GHz})$. We then save all the systems in a database that we are going to filter and rank to search for good candidates.

\subsubsection{Selection of solutions}

First we generate a collimator and an imager. For each of them we follow the same procedure:

1. We generate 10 million systems with a predetermined $y-\bar{y}$ boundary.

2. We filter them using geometrical parameters such as: volume, total track etc.

3. We filter the best $1 \%$ of the remaining set based on an optical performance function calculated from Seidel sums.

4. We use a clustering algorithm to extract solutions that are reasonably different.

5. The designer chooses the most promising starting point based on extra information such as $\frac{b}{a}$.

This clustering algorithm chosen is k-means. It is used to group the systems in clusters that have similar $y-\bar{y}$ coordinates Without this step the solutions that we would get would for most of them be similar.

\subsubsection{CodeV optimization}

We have made a CodeV optimization routine that is completely hands free. It follows this procedure :

1. Optimization of the two sub systems separately without introducing aspherization.

2. Optimization of the two sub systems together without introducing aspherization.

3. Optimization of the two sub systems together with aspherization.

This procedure can be repeated for all combinations of starting points found in the previous step. Regarding decenters all mirrors are left completely free to move, we use an extensive set of constraints generated by a custom software to prevent any obscuration and another set to insure that the system does not exceed its maximum volume. 


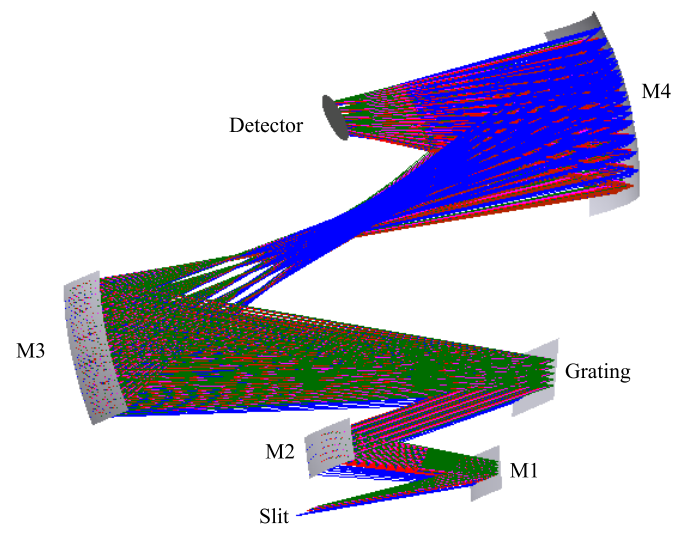

Figure 10. A TROPOLITE candidate solution. Note that there is a large available space between M4 and grating to baffle out parasitic diffraction orders coming from the grating.

\section{RESULTS}

The advantages of this method are:

- Does not require extensive experience for generating or selecting starting points.

- Makes optical designers very responsive with respect to mechanical design input.

Regarding the last point it was possible to consult with mechanical design experts and to satisfy their preferences regarding mirror locations. That way we ensure that the mirrors are mounted with minimum tolerances at a position not necessary beneficial for the nominal system but beneficial for the manufactured one.

We also suspect that this method might allow a kind of global optimization by starting the optimization with very diverse starting points. In our case the diversity of various volume and mechanical constraints meant that we had only one starting point possible which is in itself also a valuable insight.

One of the results of this study is pictured in figure 10 and 11.

\section{REFERENCES}

[1] Nijkerk, D., van Venrooy, B., van Doorn, P., Henselmans, R., Draaisma, F., and Hoogstrate, A., "The tropomi telescope," http://www.congrexprojects.com/custom/icso/2012/papers/FP_ICSO-150.pdf.

[2] Delano, E., "First-order design and the $y-\bar{y}$ diagram," Applied Optics 2(12), 1251-1256 (1963).

[3] Burch, C., "Application of the plate diagram to reflecting telescope design," Opt. Acta 26, 493-504 (1979).

[4] Lopez-Lopez, F. J., "Analytical aspects of the $y-\bar{y}$ diagram," in [Applications of Geometrical Optics II], Smith, W. J., ed., Proc. SPIE 0039, 151-166 (1974).

[5] Gross, H., Zugge, H., Peschka, M., and Blechinger, F., [Handbook of Optical Systems: Aberration Theory and Correction of Optical Systems], vol. 3, Wiley-VCH (1994). 


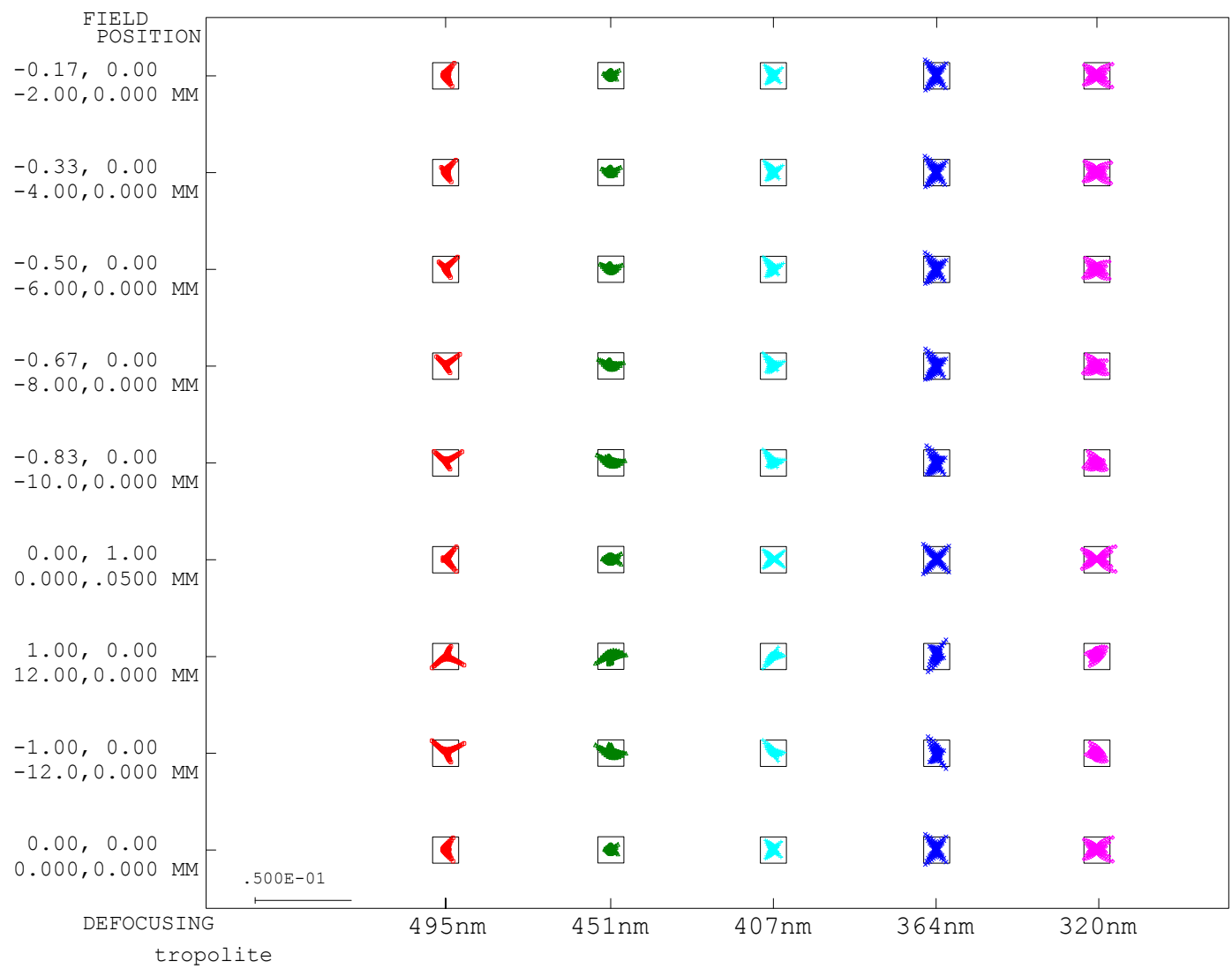

Figure 11. Spot diagrams for solution presented in figure 10. Overlay represents a $0.013 \mathrm{~mm}$ pixel.

Proc. of SPIE Vol. 9131 91310L-10 